\title{
RANDOM PHASE WAVE: A SOLUBLE NON-MARKOVIAN SYSTEM
}

R. L. DEWAR

\section{PLASMA PHYSICS LABORATORY}

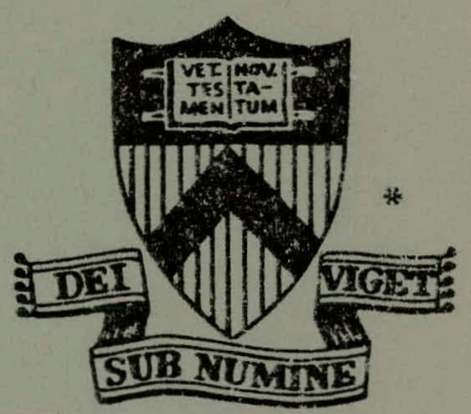

\section{PRINCETON \\ UNIVERSITY PRINCETON, NEW JERSEY}

This work was supported by Department of Energy Contract EY-76-C-02-3073. Reproduction, translation, publication, ise and disposal, in whole or in part, by or for the United Scates Government is permitted. 


\section{DISCLAIMER}

This report was prepared as an account of work sponsored by an agency of the United States Government. Neither the United States Government nor any agency Thereof, nor any of their employees, makes any warranty, express or implied, or assumes any legal liability or responsibility for the accuracy, completeness, or usefulness of any information, apparatus, product, or process disclosed, or represents that its use would not infringe privately owned rights. Reference herein to any specific commercial product, process, or service by trade name, trademark, manufacturer, or otherwise does not necessarily constitute or imply its endorsement, recommendation, or favoring by the United States Government or any agency thereof. The views and opinions of authors expressed herein do not necessarily state or reflect those of the United States Government or any agency thereof. 


\section{DISCLAIMER}

Portions of this document may be illegible in electronic image products. Images are produced from the best available original document. 
NOTICE

This report was prepared as an account of work sponsored by the United States Govermment. Neither the United States nor the United States Energy Research and Development Administration, nor any of their cmployces, nor any of their contractors, subcontractors, or their employees, makes any warranty, express or implied, or assumes any legal liability or responsibility for the accuracy, completeness of usefulness of any information, apparatus, product or process disclosed, or represents that its use would not infringe privately owned $=$ zghts.

Printed in the United states of Anerica.

Available from

NationaI Techical Information. Service

U. S. Department of Commerce 5285 Port Royal Road Springfield, Virginia 22151 Price: Printed Copy \$_*; Microfiche $\$ 3.00$

tPages

$$
1-50
$$

$51-150$

$151-325$

$326-500$

$501-1000$
NTIS

Selling Price

$\$ 4.00$

5.45

7.60

10.60

13.60 


\title{
Random Phase Wave: A Soluble Non-Markovian System
}

by

\author{
R. L. Dewar
}

Plasma Physics Laboratory, Princeton University

Princeton, New Jersey 08540

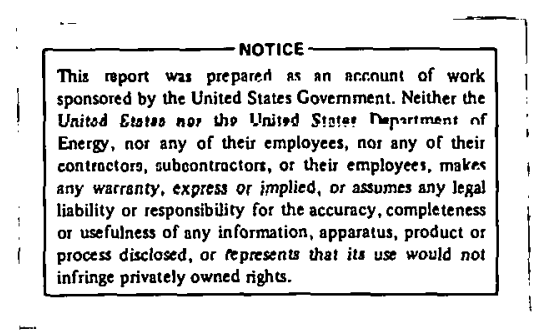


Random Phase Wave: A Soluble Non-Markovian System

R. L. Dewar ${ }^{1}$

\section{ABSTRACT}

The averaged propagator and the corresponding mass operator (non-Markovian particle-wave collision operator) of a particle being accelerated by a random potential are constructed explicitly in a model system. The model consists of an ensemble of monochromatic waves of random phase, such as arises in narrowbandwidth plasma turbulence, and is particularly interesting as a system exhibiting strong trapping. An essential simplifying feature is that the propagator is evaluated in oscillation-center picture, which greatly simplifies the momentum-space operators occurring in the problem, and leads to a remarkable factorization of the mass operator. General analyticity and symmetry properties are derived using a projection-operator method, and verified for the solution of the model system. The nature of the memory exhibited by the mass operator is briefly examined. KEY WORDS: Non-Markovian; random-acceleration; plasma turbulence; mass operator; oscillation-center.

Work commenced in Department of Theoretical Physics, Research School of Physical Sciences, The Australian National University, and completed at Princeton University under U. S. ERDA Contract F.Y $-76-C .-02-3073$.

1. Plasma Physics Laboratory, Princeton University, Princeton, New Jersey 08540 


\section{INTRODUCTION}

In a turbulent collisionless plasma a reasonable way to understand the particle dynamics is to model the plasma waves by an external random potential. Poisson's equation is applied only after all statistical averages have been taken. Although this is not fully self-consistent, it greatly simplifies the problem since only the single-particle Hamiltonian need be used.

The problem attacked in this paper is to find such a model system in which the Dyson equation for the averagedisingleparticle propagator can be constructed, and which exhibits the effects of strong particle trapping. This phenomenon occurs in narrow-bandwidth plasma turbulence. Such a system is particularly interesting because it is the case least amenable to perturbation theory. In the opposite limit of broad bandwidth, Dupree (1) showed some time ago that the Dyson equation can be approximated by a Fokker-Planck equation. This can be improved on somewhat by use of renormalized perturbation theory, the end result typically being the direct-interaction approximation, (2) which puts in some non-Markovian behavior but does not treat particle trapping. Also there is strong evidence that even the renormalized perturbation series is divergent. $(3,4)$ There is thus a real need for an analytically soluble model of the type we propose to treat.

There is also a need for general symmetry and analyticity results, such as those obtained by Forster (5) in the theory of liquids. These properties provide a test for the reasonableness 
of approximate forms of the mass operator, or a check on the correctness of exact solutions. In this paper (Appendix) we derive analogous results for the random acceleration problem using the projection operator method of Weinstock, (6) and verify that our explicit solution satisfies these requirements (Sec. 7). The simple statistical ensemble which comprises our model is presented in Sec. 2, and in sec. 3 an additional simplifying element is introduced: We make a canonical transformation to oscillation-center variables. $(7,8)$ This means that the propagator which we average is not precisely the propagator for the distribution function in the usual variables. However, because the oscillation-center transformation preserves the qualitative nature of particle orbits, we believe that the oscillation-center propagator gives an equally valid "picture" of the statistical. behavior of the system. The averaged oscillation-center propagator is calculated in Secs. 4 and 5 .

The great advantage of working in oscillation-center variables is that the momentum-space operators occurring in the problem are simplified from general Hilbert-space operators to $2 \times 2$ matrices. This enables us in Sec. 6 to work backwards from the known average propagator to the "mass operator" in the Dyson equation. An unexpected factorization of the mass operator into a simple momentum-space operator and a scalar memory function is found to occur. The general properties derived in the Appendix are verified in Sec. 7, where we also show the behavior of the memory function with respect to time delay: 


\section{STATISTICAL MODEL}

The model consists of an ensemble of one-dimensional systems in each realization of which there is a single stationary electrostatic wave present whose phase $\alpha$ is random over the ensemble. This models narrow-bandwidth plasma turbulence as seen from a frame moving with the average phase., velocity. Thus, the singleparticle Hamiltonians are of the form

$$
H_{\alpha}=\frac{p^{2}}{2 m}-e \phi_{0} \cos \left(k_{0} x-\alpha\right)
$$

where $e$ and $m$ are the particle charge and mass, respectively, and $k_{0}$ and $\phi_{0}$ are the wavenumber and amplitude (assumed to be the same in each realization). The average of any quantity $\phi_{\alpha}$ depending on $\alpha$ is

$$
\bar{\phi} \equiv\langle\phi\rangle \equiv \int_{0}^{2 \pi} \frac{\mathrm{d} \alpha}{2 \pi} \phi_{\alpha} .
$$

We can, following O'Neil, (9) find the orbits governed by Hamiltonian (1):in terms of elliptic functions. Given the orbits the exact propagator $U_{N}(\varnothing \mid 0)$ can be written down. [Notationally: we follow closely an earlier paper, (7) with $\varnothing$ denoting the unsubscripted argument list $(x, p, t)$ and 0 denoting the list $\left.\left(x_{0}, p_{0}, t_{0}\right).\right]$ The average propagator $\bar{U}(\varnothing \mid 0)$ can then be 
evaluated by applying Eq. (2), and is found to depend only on the differences $x-x_{0}$ and $t-t_{0}$, so the spatial and temporal dependence can be removed by Fourier transformation. Unfortunately, $\bar{U}_{k, \omega}\left(p, p_{0}\right)$ remains a complicated function of the initial and final momenta, thus making inversion impractical and precluding the possibility of explicitly constructing a Dyson equation for the average propagator. In order to obtain a more tractable momentum-space operator, we transform to "oscillation-center" picture.

\section{OSCILLATION-CENTER PICTURE}

In Ref. 7 it was pointed out that the propagator

$$
G_{\alpha}(\varnothing \mid 0) \equiv C_{\alpha} U_{\alpha}(\varnothing \mid 0) C_{\alpha}^{-1}
$$

with $\mathrm{C}_{\alpha}$ a unitary linear operator acting on phase space functions, is dynamically equivalent to $\mathrm{U}_{\alpha}$, being the propagator in a new set of canonical variables: the oscillation-center coordinates. Specifically, we define the oscillation-center transformation to be such that the oscillation-center momentum of an untrapped particle is a constant of the motion, while for trapped particles we require the oscillation-center momentum to be a constant of the motion almost everywhere. Because this transformation is constructed as the limiting case of a family of diffeomorphisms from phase space onto itself, ${ }^{(8)}$ the topology 
of the orbits is unchanged. Thus, the sign of the oscillationcenter momentum of a trapped particle must reverse periodically in order to keep the orbit topologically circular, the reversals being caused by collisions with thin potential barriers located at $k_{0} \mathrm{x}=(2 \mathrm{n}+: 1) \pi+\alpha$, with $\mathrm{n}$ an integer. In Ref. 8 it was shown that such a canonical transformation can indeed be constructed. The oscillation-center orbit of a trapped particle in a particular realization of the ensemble is depicted in Fig. 1 , while the graphs of position and momentum versus the dimensionless time parameter $\tau \equiv(1 / 2) \mathrm{k}_{0} \mathrm{v}_{0}\left(t-t_{0}\right)$ are shown in Fig. 2 . Here $v_{0} \equiv \partial \bar{K} / \partial p_{0}$ is the oscillation-center velocity, $K$ being the new Hamiltonian. In terms of the energy-dependent bounce frequency $\omega_{b}\left(\left|p_{o}\right| ; e \phi_{o} / m, k_{o}\right)$ we have

$$
\tau=\omega_{b}\left(p_{o}\right) \operatorname{sgn}\left(p_{o}\right)\left(t-t_{o}\right)
$$

If we denote by $\hat{x}_{\alpha}(t \mid 0)$ and $\hat{p}_{\alpha}(t \mid 0)$ the oscillation-center position and momentum of a particle as a function of its initial position $x_{0}$ and momentum $p_{0}$ at initial time $t_{0}$, the exact retarded propagator in oscillation-center picture is

$$
G_{\alpha}(\varnothing \mid 0)=\delta\left[x-\hat{x}_{\alpha}(t \mid 0)\right] \delta\left[p-\hat{p}_{\alpha}(t \mid 0)\right] \theta\left(t-t_{0}\right)
$$

where $\theta(t)$ is the Heaviside step function. 
For untrapped particles we have $\hat{p}_{\alpha}=p_{0}$ for all times. Furthermore, $\hat{x}_{\alpha}=x_{0}+v_{0}\left(t-t_{0}\right)$ is also independent of $\alpha$, so that $G_{\alpha}$ is just the unperturbed propagator for a particle with velocity $v_{0} \equiv \partial \overline{\mathrm{K}} / \partial \mathrm{p}_{0}$. As a corollary, the averaged propagator is also the unperturbed propagator. For trapped particles the propagator is not so trivial, and requires a detailed discussion of the orbit functions $\hat{x}_{\alpha}$ and $\hat{p}_{\alpha}$.

\section{ORBIT FUNCTIONS (TRAPPED PARTICLES)}

In order to simplify the analytic representation of $\hat{p}(t \mid 0)$ and $\hat{x}(t \mid 0)$ in the trapping region, we define a phase $\beta$ relative to the initial position by

$$
\beta=\pi+\alpha-k_{0} x_{0}, \operatorname{modulo} 2 \pi
$$

It is clear that the integral over $\alpha$ in Eq. (2) can be replaced by the integral from 0 to $2 \pi$ over $\beta$.

We also define a triangular waveform function, $T(\tau)$, as depicted in Fig. $3 a$. 
Both $\hat{p}$ and $\hat{d x} / d t$ can now be written in terms of the derivative $\mathrm{T}^{\prime}$, which is a square waveform. We have

$$
\hat{p}=p_{O} T^{\prime}\left(\tau-\frac{1}{2} \beta\right)
$$

and

$$
\hat{\mathrm{dx}} / \mathrm{dt}=\mathrm{v}_{\mathrm{o}} \mathrm{T}^{\prime}\left(\tau-\frac{1}{2} \beta\right)
$$

wherc, as before, $v_{0}$ is $2 \omega_{b}\left(p_{0}\right) \operatorname{sgn}\left(p_{0}\right) / k_{0}$. Integrating $E q$. we get:

$$
\hat{x}=x_{0}+2 k_{0}^{-1}\left[T\left(\tau-\frac{1}{2} \beta\right)-T\left(\frac{1}{2} \beta\right)\right] .
$$

If we denote $\tau$ modulo $2 \pi$ by $\tau^{\prime} \equiv \tau-2 \mathrm{n} \pi$, with $\mathrm{n}$ an integer chnsen so that $\tau^{\prime}$ is in the intorval $[0,2 \pi)$, then four cases may be distinguished:

$\begin{array}{rlrl}\text { Case } 1 & 0 \leq \tau^{!}<\pi, & 0 \leq \frac{1}{2} \beta<\tau^{\prime} \\ & \hat{p}=-p_{0}, & \hat{x}=x_{0}+2 k_{0}^{-1}\left(\beta-\tau{ }^{\prime}\right)\end{array}$ 
Case 2

$$
\begin{array}{ll}
0 \leq \tau^{\prime}<\pi, & \tau^{\prime} \leq \frac{1}{2} \beta<\pi \\
\hat{p}=p_{0}, & \hat{x}=x_{0}+2 k_{0}^{-1} \tau
\end{array}
$$

Case 3

$$
\pi \leq \tau^{\prime}<2 \pi, \quad 0 \leq \frac{1}{2} \beta<\tau^{\prime}-\pi
$$

$$
\hat{p}=p_{0} \quad, \quad \hat{x}=x_{0}+2 k_{0}^{-1}\left(\tau^{\prime}-2 \pi\right)
$$

Case $4 \quad \pi \leq \tau^{\prime}<2 \pi, \quad \tau^{\prime}-\pi \leq \frac{1}{2} \beta<\pi$

$$
\hat{p}=-p_{0}, \quad \hat{x}=x_{0}+2 k_{0}^{-1}\left(\beta-\tau^{\prime}\right)
$$

It is convenient to define a sawtooth waveform function $S(\tau)$ as depicted in Fig. $3 b$. In cases 2 and $3, \hat{x}$ is then simply $x_{0}+2 k_{0}^{-1} s(\tau)$. 


\section{AVERAGE PROPAGATOR}

It is now a simple matter to perform the integrations over $B$ to calculate $\bar{G}(\varnothing \mid 0)$. First observe that in cases 2 and 3 the integration is trivial because the integrand is independent of $\beta$ over the ranges indicated. In cases 1 and 4 the delta function containing $\hat{x}$ is removed by the integration, bearing in mind that there is one and only one solution of the equation $\hat{x}=x$ when $x$ is in the range such that

$$
\left|x-x_{0}\right|<2 k_{0}^{-1}|S(\tau)|
$$

Outside this range: there is no solution. Also observe that $|\partial \hat{\mathrm{x}} / \partial \beta|=2 \mathrm{k}_{0}^{-1}$ in cases 1 and 4 . The result of the averaging is thus

$\bar{G}(\varnothing \mid 0)=\pi^{-1} T(\tau) \theta\left(t-t_{0}\right) \delta\left[x-x_{0}-2 k_{0}^{-1} S(\tau)\right] \delta\left(p-p_{0}\right)$

$$
+(4 \pi)^{-1} k_{0} \theta\left(t-t_{0}\right) \theta\left(2 k_{0}^{-1}|s(\tau)|-\left|x-x_{0}\right|\right) \delta\left(p+p_{o}\right) \cdot
$$

Note that, for $t>t_{\Omega}, \bar{G}$ is a periodic function of $t$; the initial state recurring every bounce period. There is no irreversible phase mixing because $p_{0}$ defines a unique bounce frequency in oscillation-center picture (provided $k_{0}$ and $\phi_{0}$ are the same in all realizations of the ensemble). 
Note that Eq. $(10)$ is also valid for $\mathrm{p}_{0}<0$, with $\tau$ defined as in Eq. (4). Thus, Eq. (10) expresses the fact that there is a nonvanishing probability of transitions only between the states with $p= \pm\left|p_{0}\right|$. To make this "coupled level" picture more explicit, we introduce a matrix notation for $\bar{G}$, and also choose to Fourier transform $\bar{G}$ at this time:

Case A. $\quad \mathrm{p}_{0}=+\left|\mathrm{p}_{\mathrm{o}}\right|$

$$
\begin{aligned}
\bar{G}(\not \mid 0) & =\iint \frac{d k d \omega}{(2 \pi)^{2}} \exp \left[i k\left(x-x_{0}\right)-i(n)\left(t-t_{0}\right)\right] \\
& \times i\left[\delta\left(p-\left|p_{0}\right|\right) \bar{G}_{k, \omega}^{++}\left(\left|p_{0}\right|\right)+\delta\left(p+\left|p_{0}\right|\right) \bar{G}_{k, \omega}^{-+}\left(\left|p_{0}\right|\right)\right]
\end{aligned}
$$

Case B. $\quad \mathrm{p}_{\mathrm{O}}=-\left|\mathrm{p}_{\mathrm{O}}\right|$

$$
\begin{aligned}
\bar{G}(\not \mid 0)= & \iint \frac{d k d \omega}{(2 \pi)^{2}} \exp \left[i k\left(x-x_{0}\right)-i \omega\left(t-t_{0}\right)\right] \\
& \times i\left[\delta\left(p-\left|p_{o}\right|\right) \bar{G}_{k, \omega}^{+-}\left(\left|p_{o}\right|\right)+\delta\left(p+\left|p_{o}\right|\right) \bar{G}_{k, \omega}^{--}\left(\left|p_{0}\right|\right)\right]
\end{aligned}
$$


where a factor $i$ has been taken out of $\bar{G}_{k, \omega}^{\rho \sigma}$ (7) to make it asymptotically equal to $\left(\omega-k \rho v_{o}\right)^{-1} \delta_{\rho, \sigma}$ as $\operatorname{Im} \omega \rightarrow+i \infty$. If $\sigma$ denotes + or - , the diagonal matrix elements are given by

$\bar{s}_{k, \omega}^{\sigma \sigma}=\frac{1}{\omega-\sigma k v_{0}}-2 \omega_{b} \frac{\sin \left\{\left[\pi\left(\omega+k v_{o}\right) j / 2 \omega_{b}\right\} \sin \left\{\left[\pi\left(\omega-k v_{o}\right)\right] / 2 \omega_{b}\right\}\right.}{\pi \sin \left(\pi \omega / \omega_{b}\right)\left(\omega-\sigma k v_{o}\right)^{2}}$,

while the off-diagonal matrix elements are given by

$\bar{G}_{k, \omega}^{+-}=\bar{G}_{k, \omega}^{+}=2 \omega_{b} \frac{\left.\left.\sin \left\{\left[\pi(\omega)+k v_{\Omega}\right)\right] / 2(1)_{h}\right\} \sin \left\{\left[\pi(\omega)-k v_{n}\right)\right] / 2 \omega_{h}\right\}}{\| \sin \left(\| \omega / \omega_{b}\right)\left(\omega \omega^{2}=k^{2} v_{0}^{2}\right)}$.

Here $v_{0}$ denotes, $2 \omega_{b} / k_{0}$, and $\omega$ is to be interpreted as $\omega+i 0$ near zeros of the denominators.

6. DYSON EQUATION

It is shown in the appendix that a mass operator $\sum$ should exist such that $\bar{G}$ obeys the Dyson equation 


$$
\left(\partial_{\mathrm{t}}+\mathrm{L}_{\overline{\mathrm{K}}}\right) \overline{\mathrm{G}}(\varnothing \mid 0)+\int \mathrm{dl} \sum(\varnothing \mid 1) \overline{\mathrm{G}}(1 \mid 0)=\delta(\varnothing \mid 0) .
$$

For untrapped particles, $\sum$ vanishes identically, while from Eq. (11) it is clear that $\sum$ can couple only the momenta $\pm p_{0}$, so we can use a matrix representation for $\sum$ similax to that for $\bar{G}$. Using a representation for $\sum$ precisely similar to Eq. (11) for $\bar{G}$ (including the factor of $i$ ), Eq. (14) becomes

$$
\left(\mathrm{G}_{\mathrm{k}, \omega}^{(0)-1}-\sum_{\approx \mathrm{k}, \omega}\right) \underset{\approx \mathrm{G}, \omega}{\overline{\mathrm{G}}_{\mathrm{k}}}=\underset{\approx}{\mathrm{I}},
$$

with $\underset{\approx}{I}$ the unit $2 \times 2$ matrix. Here $\underset{\approx}{\bar{G}_{k}, \omega}$ is the matrix

$$
\overline{\mathrm{G}}_{\mathrm{k}, \omega} \equiv\left(\begin{array}{cc}
\overline{\mathrm{G}}_{\mathrm{k}, \omega}^{++} & \overline{\mathrm{G}}_{\mathrm{k},(1)}^{+-} \\
& \\
& \\
& \\
\overline{\mathrm{G}}_{\mathrm{k}, \omega}^{-+} & \overline{\mathrm{G}}_{\mathrm{k}, \omega}^{--}
\end{array}\right) \text {. }
$$

The inverse unperturbed propagator is given by

$$
\underset{z \mathrm{k}, \omega}{\mathrm{G}-1}=\left(\begin{array}{cc}
\omega-\mathrm{kv}_{0} & 0 \\
0 & \omega+\mathrm{kv}_{0}
\end{array}\right)
$$


Given $\bar{G}_{\mathbf{k}, \omega}$ from Eqs. (12) and (13) it is an easy matter to solve Eq. (15) for $\sum_{\approx k, \omega}$. We find that the momentum-space operator part of $\sum_{\approx k ; \omega}$ factorizes out in a very simple manner

$$
\sum_{\approx k, \omega}=\sum_{k j, \omega} \cdot\left(\begin{array}{cc}
1 & -1 \\
-1 & 1
\end{array}\right),
$$

with the scalar memory function $\sum_{k, \omega}$ being given by

$$
\sum_{k, \omega}=-2 \omega_{b}^{2} \frac{\sin \left\{\left[\pi\left(\omega+k v_{0}\right)\right] / 2 \omega_{b}\right\} \sin \left\{\left[\pi\left(\omega-k \dot{v}_{o}\right)\right] / 2 \omega_{b}\right\}}{\pi^{2} \omega F\left[\left(\pi \omega / 2 \omega_{b}\right),\left(k / k_{0}\right)\right]}
$$

The function $F$ in the denominator is defined by

$$
F(\zeta, k) \equiv \frac{\sin 2 \zeta}{2 \zeta}-\frac{\sin (\zeta+\pi k) \sin (\zeta-\pi k)}{\zeta^{2}-\pi^{2} \kappa^{2}} .
$$

In the denominator of Eq. (19), $\omega$ is to be read as $\omega_{i}+i 0$.

\section{PROPERTIES OF THE MASS OPERATOR}

(a) Symmetries

The following general symmetries are readily verified for $\operatorname{both} \overline{\mathrm{G}}_{\mathrm{k}, \omega}$ and $\sum_{\mathrm{k}, \omega}$ : 


$$
\sum_{k, \omega}\left(p_{1}, p_{2}\right)=-\sum_{-k,-\omega}^{*}\left(p_{1}, p_{2}\right)
$$

This is just the reality condition, the change of sign being a consequence of the factor of $i$ taken out in the definitions of $\bar{G}_{k, \omega}$ and $\sum_{k, \omega}[$ Eq. (11)].

$$
\sum_{k, \omega}\left(p_{1}, p_{2}\right)=\sum_{-k, \omega}\left(-p_{1},-p_{2}\right)
$$

This is a consequence of symmetry (on average) under spatial inversion. Finally, symmetry under interchange cf momenta

$$
\sum_{k, \omega}\left(p_{1}, p_{2}\right)=\sum_{k, \omega}\left(p_{2}, p_{1}\right)
$$

can be derived from time reversal and spatial inversion invariance.

There is also an antisymmetry of the matrix in Eq. (18) about a horizontal axis. This symmetry is a kind of detailed balance and ensures conservation of probability at $k=0$. 


\section{(b) Causality}

We must show that $\sum_{k, \omega}$ obeys the causality condition of being an analytic function of $\omega$ in the upper half plane. It suffices to show that $F(\zeta, k)$ has no zeros if $\zeta$ lies in the upper half plane. :

To prove this by the Nyquist technique, i.t. is suffirient. to show that the hodograph of $F(\zeta, k)$ does not encircle the origin as $\zeta$ traces out the contour shown in Fig. 4. The contour runs along the real axis from $-R$ to $+R$, except for small semicircular. sections passing above the real zeros of $F(\zeta, k)$, and then the contour returns to $(-R, 0)$ along a large semicircle in the upper half plane.

For large $|\dot{\zeta}|:, F(\zeta, k)$ can be approximated by $\sin (2 \zeta) / 2 \zeta$, so that the hodographs of $F(\zeta, k)$ and $\sin (2 \zeta) / 2 \zeta$ must be essentially identical as $\zeta$ traverses the large semicircle, looping the origin $+n$ times (say). Each time the contour crosses two consecutive zeros on the real axis, the hodographs loop the origin -l times in the contour-clockwise sense. Since $\sin (2 \zeta) / 2 \zeta$ has no zeros in the upper (or'lower) half-plane, there must be $2 n$ zeros in the interval of the real axis $(-R, R)$, so that the net number of times the hodograph encircles the origin is $-n+n=0$. To show that $F(\zeta, k)$ also has no zeros in the upper half $\zeta$ plane then, all we need 
do is to show that it has the same number $(2 n)$ of real zeros as $\sin (2 \zeta) / 2 \zeta$ in any large interval $(-R, R)$ of the real axis, so that its hodograph also loops the origin: $-n+n=0$ times.

By graphing the two terms which subtract to form $F(\zeta, k)$, it is found that the number of crossings of the two graphs (zeros of $F$ ) is the same as the number of zeros of $\sin (2 \zeta) / 2 \zeta$ in a long interval on the real line. This is found to hold for all $k$, thus proving that the causality condition is satisfied by $\sum_{k, \omega}$. In the Appendix it is shown that this is a general property, following from the anti-self-adjointness of the Liouville operator.

(c) Spectral Representation

As shown in the Appendix, anti-self-adjointness of the Liouville operator implies the existence of the spectral representation (5)

$$
\sum_{k, \omega}\left(p_{1}, p_{2}\right)=\int_{-\infty}^{\infty} \frac{d \omega^{\prime}}{2 \pi} \frac{\Gamma_{k, \omega^{\prime}}\left(p_{1}, p_{2}\right)}{\omega-\omega^{\prime}+i 0}
$$

where the damping matrix $\Gamma_{k, \omega}$ is a positive semidefinite operator. That is, 


$$
\iint \mathrm{dp}_{1} \mathrm{dp}_{2} \psi^{*}\left(\mathrm{p}_{1}\right) \Gamma_{\mathrm{k}, \omega}\left(\mathrm{p}_{1}, \mathrm{p}_{2}\right) \Psi\left(\mathrm{p}_{2}\right) \geq 0
$$

for arbitrary functions $\psi$.

In the $2 \times 2$ matrix representation of Eq. (11), we have

$$
\underset{\approx k, \omega}{\Gamma}=\Gamma_{k, \omega}\left(\begin{array}{cc}
1 & -1 \\
-1 & 1
\end{array}\right) \text {, }
$$

where the scalar damping function $\Gamma_{k, \omega}$ is given by

$$
\begin{aligned}
\Gamma_{k, \omega}= & \frac{4 \omega_{b}^{2}}{\pi}\left[\frac{\sin ^{2}(\pi k)}{F(0, k)} \delta(\omega)\right. \\
& \left.-\sum_{i=-\infty}^{\infty} \frac{\sin \left(\zeta_{i}+\pi k\right) \sin \left(\zeta_{i}-\pi k\right)}{\zeta_{i,} \partial F\left(\zeta_{i}, k\right) / \partial \zeta_{i}} \delta\left(\omega-\frac{2 \zeta_{i} \omega_{b}}{\pi}\right)\right]
\end{aligned}
$$

where $k \equiv k / k_{0}$ and the $\zeta_{i}$ are the zeros of $F(\zeta, k)$. The matrix part of Eq. (26) is positive-semidefinite [with a null vector $(1,1)]$, so one simply has to show that the scalar $\Gamma_{k, \omega}$ is $\geq 0$. One finds that the coefficients of the $\delta$ functions are always positive, so the requirement is satisfied. 
Note that $\Gamma_{\mathbf{k}, \omega}$ does not go to zero as $|\omega| \rightarrow \infty$, the asymptotic behavior being a "row of $\delta$ functions" with weights alternating between two different positive constants.

(d) Temporal Behavior

Inverting the Fourier transform of the memory function, we find its temporal behavior

$$
\begin{aligned}
\sum_{k}(t) & =\int_{-\infty}^{\infty} \frac{d \omega}{2 \pi} i \sum_{k, \omega} e^{-i \omega t} \\
& =\theta(t) \int_{-\omega}^{\infty} \frac{d \omega}{2 \pi} \Gamma_{k, \omega} e^{-i \omega t} .
\end{aligned}
$$

The function $\sum_{k}(t)$ is graphed in Fig. 5 for the case $k=0.2 k_{0}$. It is seen that the asymptotic behavior of $\Gamma_{k, w}$ at large $\omega$ leads to the occurrence of a $\delta$ function spike at time $t=0$, as if there were a component of the spectrum with zero autocorrelation time. However, these spikes recur periodically at later times, and there is also a piecewisecontinuous aperiodic component. 


\section{DISCUSSION}

The two most striking features of the oscillation-center mass operator are the separation of the mass operator into a product of a scalar memory function and a momentum-space transition probability matrix, and the occurrence of $\delta$ functions in the temporal behavior of $\sum_{k}(t)$. Because the Hamiltorian system is integrable in each realization of the ensemble, the only randomness in the problem is that introduced by the ensemble. In more: typical (and less tractable) cases the particle motion itself is highly unpredictable in most regions of phase space, thus damping the quasiperiodic tail of $\sum_{k}(t)$. It is hoped that similar simplifications will be achieved when the oscillation-center transformation is applied to such nonintegrable systems. To treat such problems, it will be necessary to develop a convergent perturbation theory for the Hamilton-Jacobi equation for the Lie generating function. (8)

It might be taken as an objection to the use of the oscillation-center transformation to simplify the propagator that we end up calculating the propagator for the average oscillation-center distribution function rather than that for the average of the true distribution function. However, because the nature of the transformation is such that the discrepancy between the oscillation-center orbit and the corresponding exact orbit has no secular component, the long-time behavior of the two propagators must be similar. This would be especially true in 
systems where there are no trapped particles since the ratio of the distance between the exact and oscillation-center positions to the displacement from the initial point tends to zero at large times in such systems. The average distribution functions do differ by a nonsecular component related to the "fake diffusion" occurring in the conventional approach, (10) but the information contained in this component can be obtained from the known transformation operator $C_{W}$, which gives the coherent response of the plasma to the potential. It is in fact an advantage of the oscillation-center approach that this coherent response is separated from the secular behavior.

\section{ACKNOWLEDGMENTS}

It is a pleasure to acknowledge useful conversations with Drs. J. A. Krommes, I. Lerche, and J. M. Greene. 


\section{APPENDIX}

In order to apply the projection operator ${ }^{(6)}$ technique, we define operators on the space of functions of $x, p$, and $\alpha$. The inner product in this space is defined by

$$
\langle f, g\rangle \equiv \iint d x d p \int_{0}^{2 \pi} d \alpha f_{\alpha}^{*}(x, p) g_{\alpha}(x, p):
$$

We now define the averaging operator $A$ such that

$$
\langle\mathrm{f}, \dot{A g}\rangle \equiv \iint \mathrm{dx} d p \int_{0}^{2 \pi} d \alpha \int_{0}^{2 \pi} \frac{d \alpha}{2 \pi} f_{\alpha}^{*}(x, p) g_{\alpha^{\prime}}^{\prime}(x, p) \text {, }
$$

for all $\mathrm{f}$ and $\mathrm{g}$. Clearly, $\mathrm{A}$ is a self-adjoint projection operator. We also define the complementary projector B by

$$
\mathrm{B} \equiv 1-\mathrm{A} \text {. }
$$

For time-independent systems, such as the one studied in this paper, the: equation for the Fourier-transformed exact propagator $\hat{G}_{\omega}$ (regarded as an operator in the space defined in this Appendix) is

$$
(\omega+i \bar{L}) \hat{G}_{\omega}+i \tilde{L}_{\omega}=1 \text {, }
$$

where $\overline{\mathrm{L}}$ is the average part $\mathrm{L}_{\overline{\mathrm{K}}}$ of the Liouville operator and $\tilde{\mathrm{L}}$ 
is the residual part $\mathrm{L}_{\tilde{\mathrm{K}}}$. Provided the space of functions of $x$ and $p$ is suitably defined, $i \bar{L}$ and $i \tilde{L}$ are self-adjoint operators. By multiplying Eq. (A4) on the left by $A$ and on the right by $A$ and $B$ we obtain two coupled equations for $\hat{A G A}$ and $\hat{B G A}$. Eliminating the latter we have

$$
(\omega+i \bar{L}) \hat{A G}_{\omega} A+A \tilde{L}\left(\omega+i \bar{L}+i \tilde{L}_{B}\right)^{-1} \tilde{L}_{\mathcal{A}} \hat{G}_{\omega} A=1,
$$

where we have used the fact that $\tilde{A L A}=0$.

It is clearly consistent with Eq. (2) to define $\bar{G}_{k, \omega}$ by

$$
2 \pi \delta\left(k-k^{\prime}\right) \bar{G}_{k, \omega}\left(p, p^{\prime}\right)=\left\langle k, p\left|\hat{G}_{\omega}\right| k^{\prime}, p^{\prime}\right\rangle
$$

where the kets $\mid \mathrm{k}^{\prime}, \mathrm{p}^{\prime}>$ are defined componentwise by

$$
\left\langle x, p, i x \mid k^{\prime}, p^{\prime}\right\rangle \equiv(2 \pi)^{-1 / 2} e^{i k k^{\prime} x} \delta\left(p-p^{\prime}\right)
$$

Note that: $A|k, p\rangle=|k, p\rangle$. 'lhus, $E q$. (A5) becomes

$$
\begin{aligned}
& (\omega-k v) \bar{G}_{k, \omega}\left(p, p^{\prime}\right)-\int d p^{\prime} \sum_{k, \omega}\left(p, p^{\prime \prime}\right) \bar{G}_{k, \omega}\left(p^{\prime}{ }^{\prime}, p^{\prime}\right) \\
& =\delta\left(p-p^{\prime}\right)
\end{aligned}
$$

where $v \equiv \partial \overline{\mathrm{K}} / \partial \mathrm{p}$ and 
$2 \pi \delta\left(k-k^{\prime}\right) \sum_{k, \omega}\left(p, p^{\prime}\right)=\left\langle k, p\left|\tilde{L}^{\dagger}(\omega+i \bar{L}+i B \tilde{L} B)-I_{\tilde{L}}^{\sim}\right| k^{\prime}, p^{\prime}\right\rangle$,

$\tilde{\mathrm{L}}^{\dagger}(=-\tilde{\mathrm{L}})$ being the adjoint of $\tilde{\mathrm{L}}$.

Since $\overline{\mathrm{L}}+\hat{\mathrm{BL} B}$ is anti-self-adjoint, it can be written in

the spectral representation (1l)

$$
\overline{\mathrm{L}}+\tilde{\mathrm{BLB}}=\mathbf{i} \int_{-\infty}^{\infty} \lambda \mathrm{dE}(\lambda)
$$

where the projection operators $E(\lambda)$ are the spectral family of $\overline{\mathrm{L}}+\mathrm{B} \tilde{\mathrm{L}} \mathrm{B}$, and the integral runs over real $\lambda$. In this representation Eq. (A9) becomes

$2 \pi \delta\left(k-k^{\prime}\right) \sum_{k, \omega}\left(p, p^{\prime}\right)=\int_{-\omega}^{\infty} d \lambda \frac{d<k, p\left|\ddot{L}^{\dagger} E(\lambda) \tilde{L}\right| k^{\prime}, p^{\prime}>/ d \lambda}{0-\lambda+\dot{i} 0}$.

Thus the existence of Eq. (24) is guaranteed, and hence so is the causality property. Also, from the definition of $E_{i}(\lambda)(11)$ it is clear that $\sum_{k, \omega}$ is a positive semi-definite operator. 


\section{REFERENCES}

1. T. H. Dupree, Phys. Fluids $\underline{9}: 1773$ (1966).

2. S. A. Orszag and R. H. Kraichnan, Phys. Fluids 10:1720 (1967) .

3. S. A. Orszag, Phys. Fluids 18:271 (1975).

4. I. G. Enting, Phys. Letters 59A:417 (1977).

5. D. Forster, Phys. Rev. A $\underline{9}: 943$ (1974).

6. J. Weinstock, Phys. Fluids 12:1045 (1969).

7. R. L. Dewar, J. Phys. A 9:2043 (1976).

8. R. L. Dewar, J. Phys. A (to be published).

9. T. M. O'Neil, Phys. Fluids 8:2255 (1965).

10. R. L. Dewar, Phys. Fluids 16:1102 (1973).

11. P. Roman, Some Modern Mathematics for Physicists and Other Outsiders, (Pergamon, New York, 1975) Vol. 2, p. 633 . 


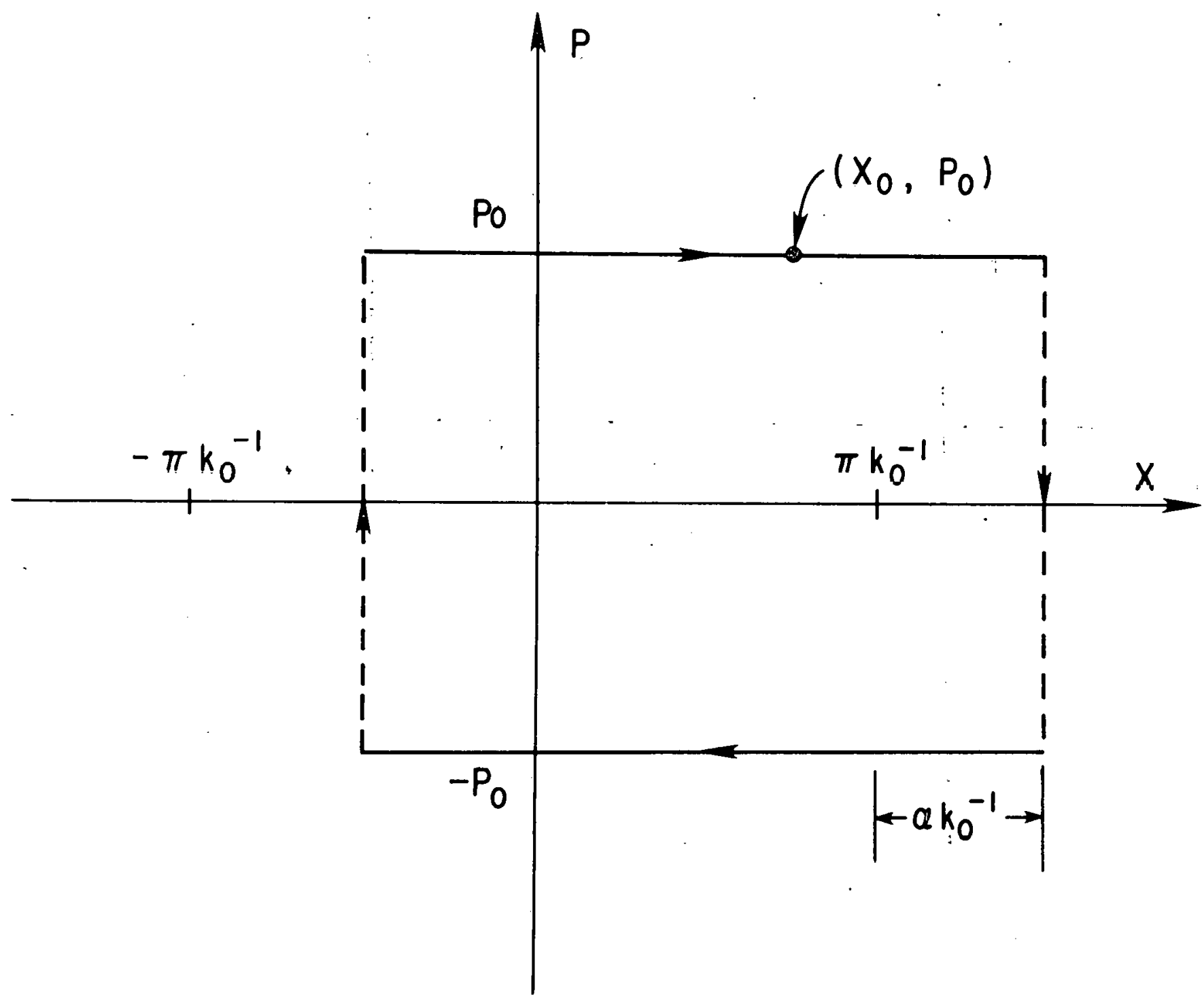

Fig. 1. The orbit $x=\hat{x}_{\alpha}(t \mid 0), p=\hat{p}_{\alpha}(t \mid 0)$ of a trapped particle, in oscillation-center picture. 


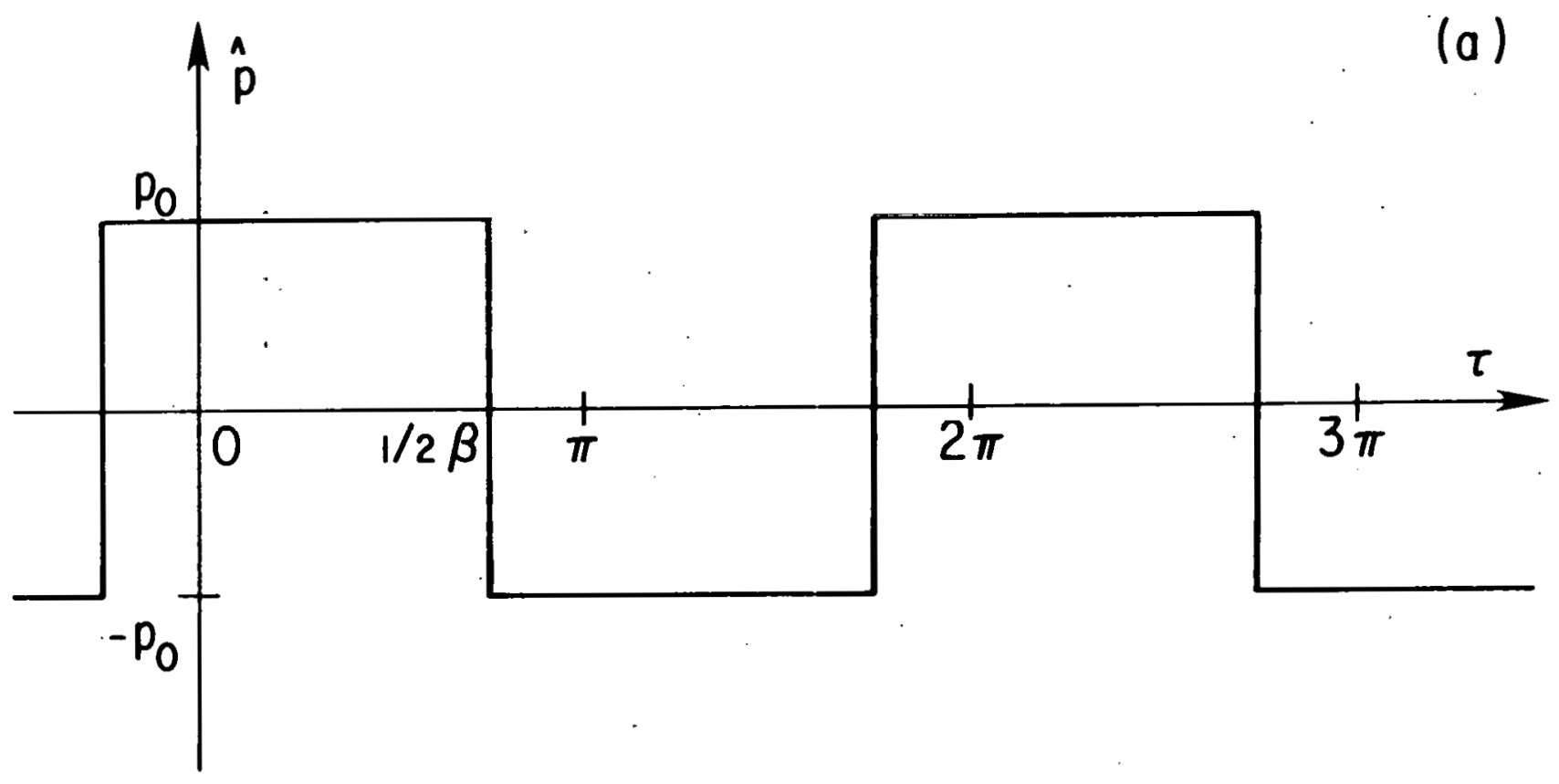

(b)

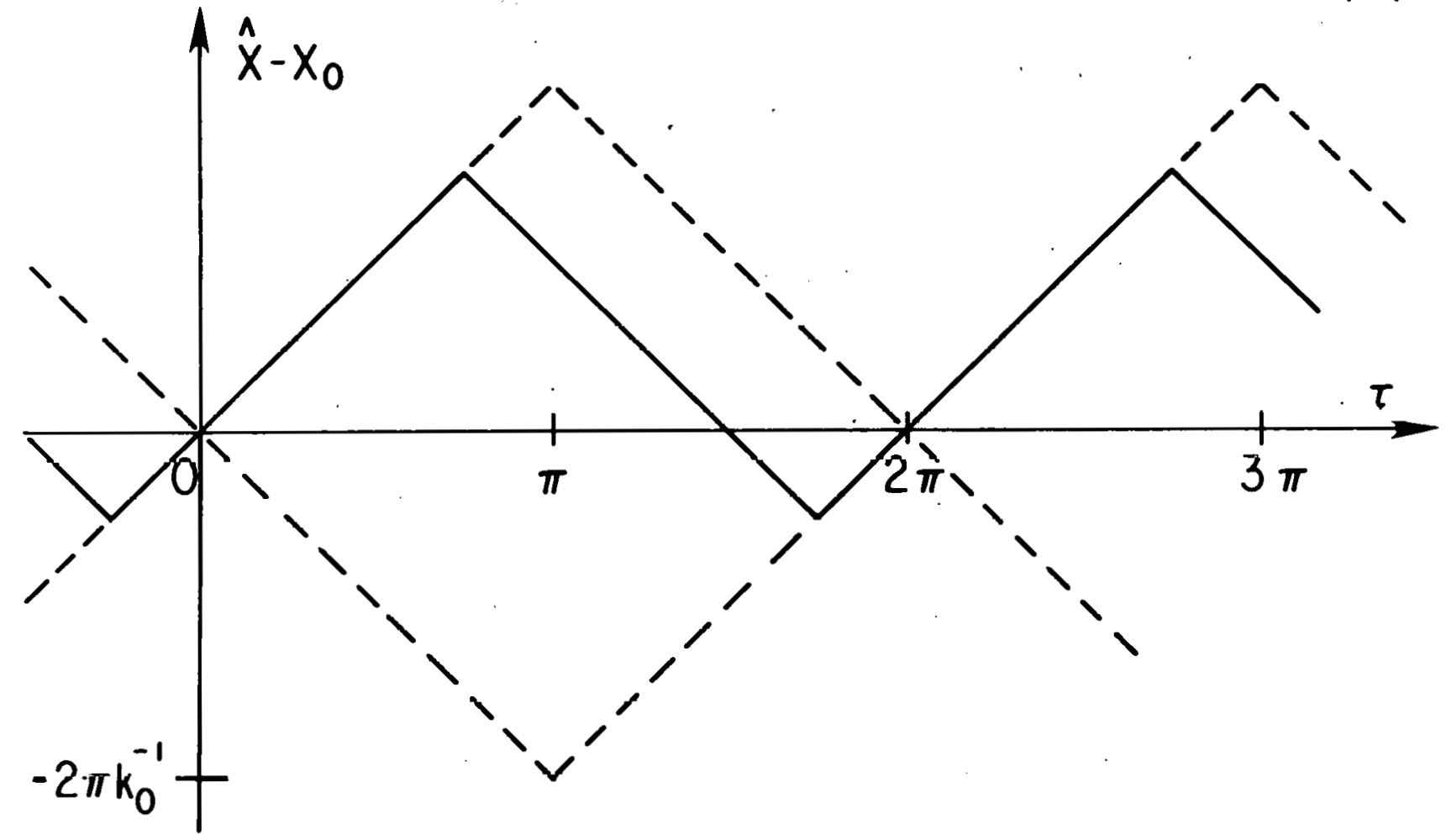

772.508

Fig. 2. Graphs of the functions $\hat{p}_{\alpha}(t \mid 0)$ and $\left[\hat{x}_{\alpha}(t \mid 0)-x_{0}\right]$ vs $\tau$. The dashed lines in Fig. 2b are the extreme cases when $x_{0}$ 1s just to the right or left of a potential barrier. 

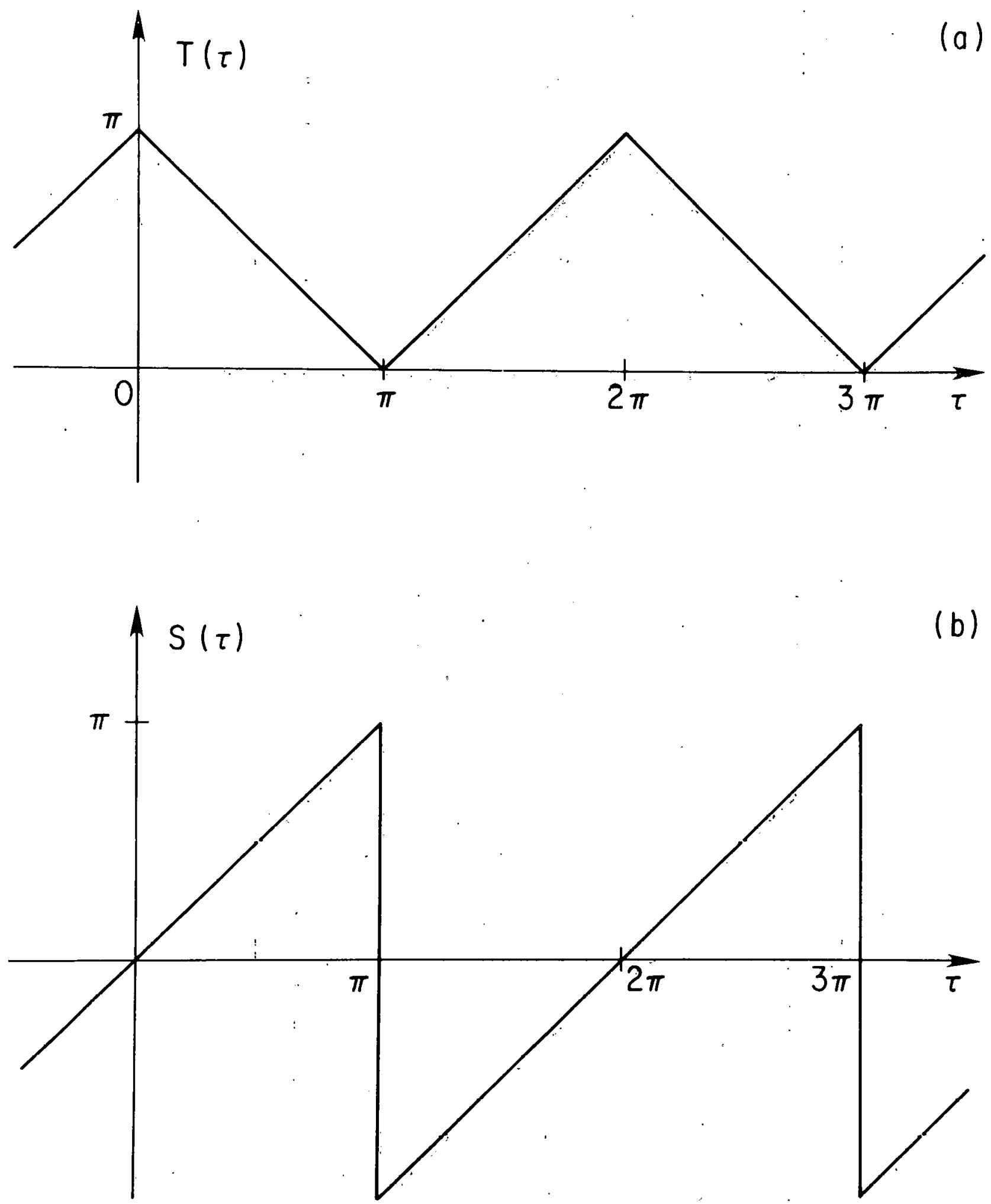

772503

Fig. 3. The periodic triangular and sawtooth waveforms $T(\tau)$ and $S(\tau)$ used in the analytic representation of the orbits. 


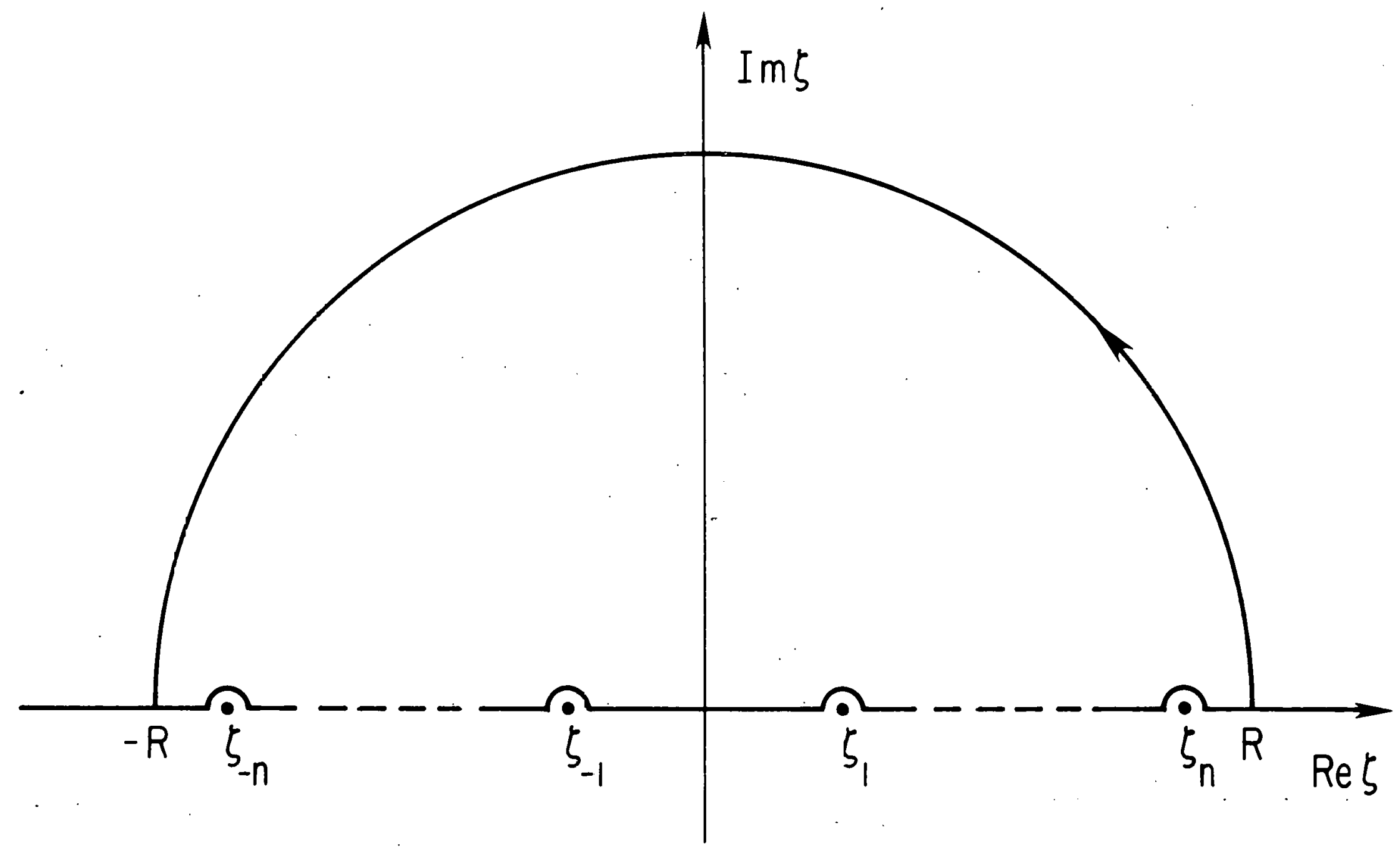

Fig. 4. The Nyquist contour used for showing that the zeros, $\zeta_{i}$, of $F(\zeta, k)$ are all on the real $\zeta$ axis. 


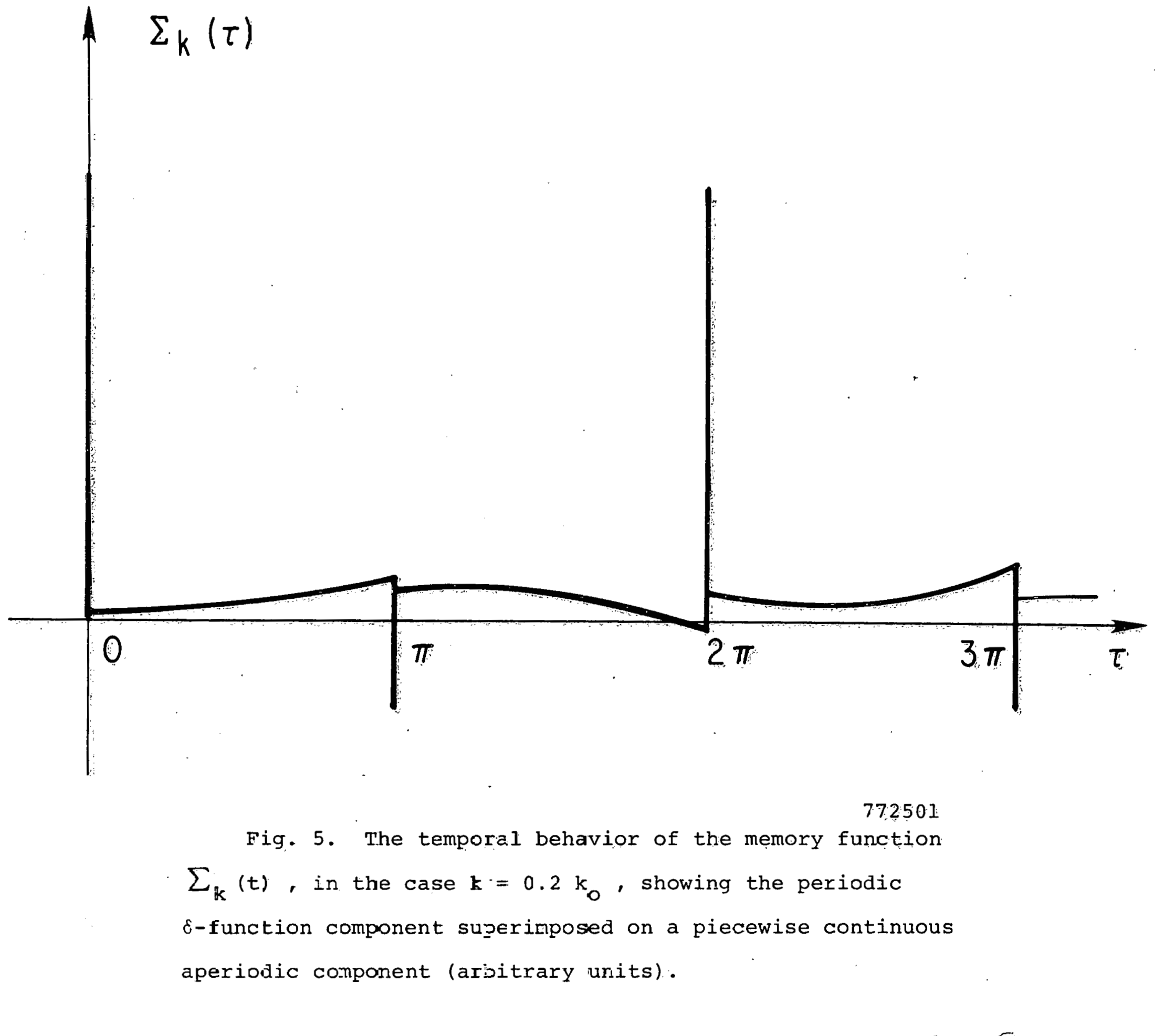

$\stackrel{1}{\stackrel{1}{~}}$ 